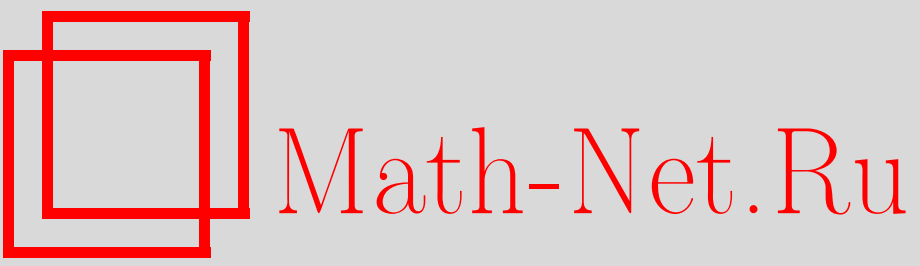

П. В. Гапеев, Расчет верхних и нижних цен опционов Европейского типа, УМH, 1997, том 52, выпуск 4, 199-200

DOI: https://doi.org/10.4213/rm868

Использование Общероссийского математического портала Math-Net.Ru подразумевает, что вы прочитали и согласны с пользовательским соглашением

http://www.mathnet.ru/rus/agreement

Параметры загрузки:

IP : 107.22 .136 .117

26 апреля 2023 г., 18:17:03 


\title{
РАСЧЕТ ВЕРХНИХ И НИЖНИХ ЦЕН ОПЦИОНОВ ЕВРОПЕЙСКОГО ТИПА
}

\author{
П. В. Г АПЕЕВ
}

В работе А. Н. Ширяева, Ю. М. Кабанова, Д. О. Крамкова и А. В. Мелшникова [1] была рассмотрена модель Кокса-Росса-Рубинштейна, где $(B, S)$-рынок предполагался полным. Было дано определение рациональной стоимости опциона Европейского типа.

Мы рассмотрим вопрос о том, как надо определять понятия цены покупки и цены продажи опциона в схеме, где активный трейдер может не только продавать, но и покупать опционы Европейского типа, при этом совершая хеджирование того и другого. Рассматривается модель неполного рынка, где множество мартингальных мер состоит более чем из одной меры.

Пусть имеется дискретньй $(B, S)$-рьнок. Стоимость акции $S=\left(S_{k}\right)_{k \in \mathbb{N} \cup\{0\}}$ в момент $k$ : $S_{k}=S_{0}\left(1+\rho_{1}\right)\left(1+\rho_{2}\right) \cdots\left(1+\rho_{k}\right), S_{0}>0$, где $\rho_{k}=\rho_{k}(\omega), k \in \mathbb{N},-$ независимые одинаково распределенные случайные величины, зависящие от случая $\omega \in \Omega$.

Поскольку в этой модели вся случайность входит через значения $\rho_{k}, k \in \mathbb{N}$, то можно оперировать лишь с распределением вероятности $\mathrm{P}=\mathrm{P}(d \rho)=\mathrm{P}\left(d \rho_{1} \times d \rho_{2} \times \cdots \times d \rho_{N}\right)$ на множестве $\mathbb{R}^{N}$ с борелевской системой $\mathscr{B}\left(\mathbb{R}^{N}\right)$, где $N$ - maturity time (время предъявления) опциона Европейского типа. Будем также предполагать,что носитель меры $\mathrm{P}(d \rho)$ сосредоточен на брусе $[a, b]^{N}$, $-1<a<b<+\infty$

Мы предположим, что банковский счет $B=\left(B_{k}\right)_{k \in \mathbb{N} \cup\{0\}}$ имеет ставку $r \geqslant 0,-1<a<r<b$, и $B_{k}=B_{0}(1+r)^{k}, B_{0}=1$.

Введем класс $\mathscr{P}(\mathrm{P})=\{\widetilde{\mathrm{P}}=\widetilde{\mathrm{P}}(d \rho)\}$ распределений на $[a, b]^{N}$, обладающих следующими свойствами:

$1^{\circ}$. $\widetilde{\mathrm{P}} \sim \mathrm{P}$ (т.е. меры $\mathrm{P}$ и $\widetilde{\mathrm{P}}$ взаимно абсолютно непрерьвны: $\widetilde{\mathrm{P}} \ll \mathrm{P}, \mathrm{P} \ll \widetilde{\mathrm{P}}$ );

$2^{\circ} \cdot \int_{a}^{b} \rho \widetilde{\mathrm{P}}(d \rho)=r ; k \in \mathbb{N}, k \leqslant N$.

Будем предполагать, что $\mathscr{P}(\mathrm{P}) \neq \varnothing$.

Отметим следущее обстоятельство: если мера $\widetilde{P} \sim \mathrm{P}$, то условие "Р-п.н." может быть заменено на условие "Р-п.н.".

Положим $\mathscr{F}_{0}=\left\{\varnothing, \mathbb{R}^{N}\right\}, \mathscr{F}_{1}=\sigma\left(\rho_{1}\right), \ldots, \mathscr{F}_{N}=\sigma\left(\rho_{1}, \ldots, \rho_{N}\right) ; \mathscr{F}_{0} \subseteq \mathscr{F}_{1} \subseteq \ldots \subseteq$ $\mathscr{F}_{N} \subseteq \mathscr{F}$ - поток вложенных $\sigma$-алгебр.

Таким образом, свойство $2^{\circ}$ имеет место тогда и только тогда, когда $\mathrm{E}_{\widetilde{\mathrm{P}}}\left(\frac{1+\rho_{k}}{1+r} \mid \mathscr{F}_{k-1}\right)=1$ ( $\widetilde{\mathrm{P}}$-п.н.); $k \in \mathbb{N}, k \leqslant N$, и, значит,

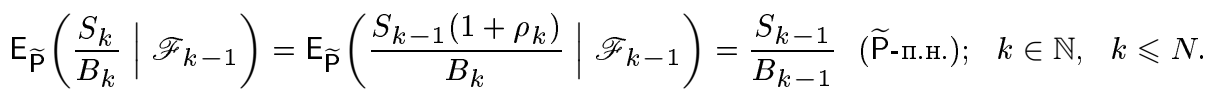

Следовательно, относительно меры $\widetilde{\mathrm{P}}$ последовательность $\left(\frac{S_{k}}{B_{k}}\right)_{k \in \mathbb{N}, k \leqslant N}$ является мартингалом.

Сделаем два предположения относителшно множества мартингалшных мер $\mathscr{P}(\mathrm{P})$.

$\left(\mathrm{A}^{*}\right)$ : существует подпоследовательность мер $\widetilde{\mathrm{P}}_{n}, n \in \mathbb{N}$, из $\mathscr{P}(\mathrm{P})$, слабо сходящаяся к меpe $\mathrm{P}^{*}$, носитель которой расположен на двух брусах размерности $N-1: \rho_{N}=a$ и $\rho_{N}=b$, где $a \leqslant \rho_{k} \leqslant b, k=1, \ldots, N-1$.

Если это предположение вьполнено, то тогда из равенств $\mathrm{E}_{\widetilde{\mathrm{P}}_{n}}\left(\frac{1+\rho_{N}}{1+r}\right)=1\left(\widetilde{\mathrm{P}}_{\text {-п.н. }}\right), n \in \mathbb{N}$, получим $\mathrm{E}_{\mathrm{P} *}\left(\frac{1+\rho_{N}}{1+r}\right)=1$ ( $\mathrm{P}^{*}$-п.н.). Отсюда находим, что

$$
p^{*}=\mathrm{P}^{*}\left(\rho_{N}=b\right)=\frac{r-a}{b-a} ; \quad 1-p^{*}=\mathrm{P}^{*}\left(\rho_{N}=a\right)=\frac{b-r}{b-a} .
$$

$\left(\mathrm{A}_{*}\right)$ : существует подпоследователшность мер $\overline{\mathrm{P}}_{n}, n \in \mathbb{N}$, из $\mathscr{P}(\mathrm{P})$, слабо сходящаяся к меpe $\mathrm{P}_{*}$, носитель которой расположен на брусе размерности $N-1: \rho_{N}=r, a \leqslant \rho_{k} \leqslant b$, $k=1, \ldots, N-1$. 
Верхней ценой (ценой продажи опциона активным трейдером, которую могут принять обе стороны) можно считать верхнюю грань тех цен, которые гарантировали бы покупателю выполнение продавцом оговариваемых условий. С другой стороны, эти цены (стоимости, премии) не должны давать продавцу "free lunch":

$$
\begin{gathered}
\mathbb{C}^{*}(\mathrm{P})=\inf _{\pi=(\beta, \gamma) \in \mathscr{H}^{*}(\mathrm{P})}\left(\beta_{0}+\gamma_{0} S_{0}\right) ; \\
\mathscr{H}^{*}(\mathrm{P})=\left\{\pi=(\beta, \gamma): \beta_{N}(1+r)^{N}+\gamma_{N} S_{N} \geqslant f\left(S_{N}\right)(\text { Р-п.н. })\right\},
\end{gathered}
$$

т.е. стратегия $\pi=(\beta, \gamma)$ является хеджирующей.

Определим понятие нижней цены (цены покупки опциона активным трейдером). Доход $f\left(S_{N}\right)$ от опциона должен быть не меньше величины $\beta_{N}(1+r)^{N}+\gamma_{N} S_{N}$. Таким образом, цена покупки

$$
\begin{gathered}
\mathbb{C}_{*}(\mathrm{P})=\sup _{\pi=(\beta, \gamma) \in \mathscr{H}_{*}(\mathrm{P})}\left(\beta_{0}+\gamma_{0} S_{0}\right) \\
\left.\mathscr{H}_{*}(\mathrm{P})=\left\{\pi=(\beta, \gamma): \beta_{N}(1+r)^{N}+\gamma_{N} S_{N} \leqslant f\left(S_{N}\right) \text { (Р-п.н. }\right)\right\} .
\end{gathered}
$$

Далее, предположим, что стратегия $\pi=(\beta, \gamma)$ обладает свойством самофинансируемости, т.е. $\beta_{n}(1+r)^{n}+\gamma_{n} S_{n}=\beta_{n-1}(1+r)^{n}+\gamma_{n-1} S_{n} \Longleftrightarrow(1+r)^{n} \Delta \beta_{n}+S_{n} \Delta \gamma_{n}=0 ; n \in \mathbb{N}$, $n \leqslant N$.

ТЕОРема 1. Пусть функция платежкного обязательств а $f\left(S_{0}\left(1+\rho_{1}\right)\left(1+\rho_{2}\right) \cdots\left(1+\rho_{N}\right)\right)$ является непрерывной и выпуклой вниз по $\rho_{N}, a \leqslant \rho_{N} \leqslant b$, и выполнено условие слабой компактности $\left(\mathrm{A}^{*}\right)$. Тогда верхняя цена

$$
\begin{aligned}
\mathbb{C}^{*}(\mathrm{P}) & =\sup _{\widetilde{\mathrm{P}} \in \mathscr{P}(\mathrm{P})} \mathrm{E}_{\widetilde{\mathrm{P}}} \frac{f\left(S_{N}\right)}{(1+r)^{N}} \\
& =p^{*} \frac{\mathrm{E}_{\mathrm{P} * f\left(S_{N-1}(1+b)\right)}}{(1+r)^{N}}+\left(1-p^{*}\right) \frac{\mathrm{E}_{\mathrm{P} * f\left(S_{N-1}(1+a)\right)}}{(1+r)^{N}} \quad\left(\mathrm{P}^{*}-\text { n.н. }\right) .
\end{aligned}
$$

ТЕОРема 2. Пусть функция платежного обязательств а $f\left(S_{0}\left(1+\rho_{1}\right)\left(1+\rho_{2}\right) \cdots\left(1+\rho_{N}\right)\right)$ является непрерывной и выпуклой вниз по $\rho_{N}, a \leqslant \rho_{N} \leqslant b$, и выполнено условие слабой компактности $\left(\mathrm{A}_{*}\right)$. Тогда нижняя цена

$$
\mathbb{C}_{*}(\mathrm{P})=\inf _{\overline{\mathrm{P}} \in \mathscr{P}(\mathrm{P})} \mathrm{E}_{\overline{\mathrm{P}}} \frac{f\left(S_{N}\right)}{(1+r)^{N}}=\frac{\mathrm{E}_{\mathrm{P}_{*}} f\left(S_{N-1}(1+r)\right)}{(1+r)^{N}} \quad\left(\mathrm{P}_{*}-\text { n.н. }\right) .
$$

\section{СПИСОК ЛИТЕРАТУРЫ}

[1] Ширяев А. Н., Кабанов Ю. М., Крамков Д. О., Мельников А. В. // Теория вероятн. и ее примен. 1994. Т. 39. № 1. С. 23-79.

Московский государственньй

Принято редколлегией университет им. М. В. Ломоносова 